\title{
Estimación de los modelos TAR cuando el proceso del ruido sigue una distribución $t$
}

\author{
Estimation of TAR Models when the Noise Process Follows a $t$ \\ Distribution
}

Hanwen Zhanga

hanwenzhang@usantotomas.edu.co

\begin{abstract}
Resumen
En este artículo se consideran los modelos TAR cuando el proceso de ruido sigue una distribución $t$. Se desarrolla un procedimiento bayesiano para la estimación de estos modelos cuando el modelo está identificado, es decir, cuando se conocen los parámetros estructurales. El método consiste en encontrar las densidades condicionales a posteriori de los parámetros e implementar un muestreador de Gibbs. Mediante un ejemplo simulado se encuentra que con esta metodología se logran buenas estimaciones de los parámetros.
\end{abstract}

Palabras clave: modelos TAR, ruido $t$, estimación bayesiana, muestreador de Gibbs.

\begin{abstract}
In this paper, TAR models with $t$ noise is considered. A Bayesian procedure for estimation is developed when the model has been identified, that is, the structural parameters have been estimated. The method consists in finding the conditional posterior densities and use the Gibbs sampler. Simulated example shows that this methodology leads good estimates of the parameters.
\end{abstract}

Key words: TAR models, $t$ noise, Bayesian estimation, Gibbs sampler.

\section{Introducción}

Los modelos TAR (Threshold Autoregressive Models) constituyen una rama de los modelos no lineales de series de tiempo, y fueron propuestos inicialmente por

${ }^{a}$ Docente investigadora. Universidad Santo Tomás. 
Tong (1978). Estos modelos, una vez identificados y estimados, tienen una interpretación simple y atractiva en comparación con otros muchos modelos no lineales. Estos modelos asumen que los valores de un proceso (denominado el proceso de umbrales) $\left\{Z_{t}\right\}$ determinan la dinámica del proceso de interés $\left\{X_{t}\right\}$. Cuando el proceso de umbrales es el mismo proceso de interés rezagado, es decir, cuando $Z_{t}=X_{t-d}$ para algún entero positivo $d$, el modelo se conoce como los modelos SETAR (Self-exciting TAR models). Tsay (1998) extendió los modelos SETAR univariados al ámbito multivariado. Nieto (2005) diseñó una metodología bayesiana para la identificación y la estimación de los modelos TAR. Por otro lado, Nieto (2008) caracterizó los modelos TAR univariados en términos de la media, varianza, media condicional y varianza condicional, y concluyó que la varianza condicional en estos modelos no es constante, de donde se puede pensar a los modelos TAR como una alternativa a los modelos GARCH para describir la heterocedasticidad en los datos. Moreno (2010) comparó el ajuste de los modelos TAR con ruidos gaussianos siguiendo la metodología de Nieto (2005) con los modelos GARCH con ruidos $t$ en series financieras de indicadores de bolsas de valores, y encontró que los modelos TAR con ruidos gaussianos pueden tener dificultades para capturar la heterocedasticidad marginal de la serie y no se ajustan tan bien como los modelos GARCH.

En este artículo, se presenta una metodología bayesiana para estimar los parámetros de un modelo TAR cuando el proceso del ruido sigue una distribución $t$ donde los parámetros estructurales se asumen conocidos. Se encuentran las distribuciones condicionales a posteriori de los parámetros para ejecutar el muestreador de Gibbs. La eficiencia del método se ilustra empíricamente por medio de un ejemplo simulado.

El artículo está organizado de la siguiente forma: en la sección 2 se introduce la formulación de un modelo TAR con ruido $t$ y se encuentra su función de verosimilitud; en la sección 3 se encuentran las densidades condicionales a posteriori de los parámetros de interés y se describe la implementación del muestreador de Gibbs; en la sección 4 se presenta el ejemplo simulado para ilustrar el empeño del método; y finalmente en la sección 5 se encuentra la discusión y futuras investigaciones.

\section{Modelo TAR con ruido $t$}

\subsection{Formulación del modelo TAR}

La formulación del modelo TAR con ruido $t$ está dada por

$$
X_{t}=a_{0}^{(j)}+\sum_{i=1}^{k_{j}} a_{i}^{(j)} X_{t-i}+h^{(j)} e_{t}
$$

si $Z_{t} \in R_{j}=\left(r_{j-1}, r_{j}\right]$ para algún $j=1, \cdots, l, r_{0}=-\infty, r_{l}=\infty$. Estos valores $r_{1}<\cdots<r_{l-1}$ denominan los umbrales y definen $l$ regímenes para el modelo. 
El proceso $\left\{Z_{t}\right\}$ se denomina el proceso de umbrales, cuyo comportamiento estocástico es descrito por una cadena de Markov de orden $p$. Los valores $k_{1}, \cdots, k_{l}$ son números enteros positivos que representan los órdenes autorregresivos en los $l$ regímenes. El proceso del ruido $\left\{e_{t}\right\} \sim_{i i d} t_{n}$ es independiente del proceso de umbrales $\left\{Z_{t}\right\}$

Los parámetros del anterior modelo TAR se pueden dividir en dos grupos:

- Parámetros estructurales: el número de regímenes $l$, los $l-1$ umbrales que definen los $l$ regímenes $r_{1}, \cdots, r_{l-1}$ y los órdenes autorregresivos en los $l$ regímenes $k_{1}, \cdots, k_{l}$.

- Parámetros no estructurales: los coeficientes autorregresivos $a_{i}^{(j)}$ con $j=$ $1, \cdots, l$ y $i=0, \cdots, k_{j}$, las ponderaciones de la varianza $h^{(1)}, \cdots, h^{(l)}$ y el grado de libertad del proceso del ruido $n$.

En este artículo, se asume que los parámetros estructurales son conocidos, de manera que el experto en el tema puede tener cierto conocimiento acerca de la dinámica y dar valores a los parámetros estructurales, como también estos parámetros estructurales pueden ser encontrados mediante métodos empíricos o con el criterio de selección de NAIC.

\subsection{Función de verosimilitud del modelo}

Una de las herramientas fundamentales en el modelamiento es la función de verosimilitud. A continuación se halla esta función usando el mismo procedimiento en Nieto (2005), pero adoptando la distribución $t_{n}$ del proceso del ruido.

Condicionando en los parámetros estructurales $l, r_{1}, \cdots, r_{l-1}, k_{1}, \cdots, k_{l}$ y los valores iniciales $\mathbf{x}_{k}=\left(x_{1}, \cdots, x_{k}\right)$, y denotando $k=\operatorname{máx}\left\{k_{1}, \cdots, k_{l}\right\}$, la función de verosimilitud de las series observadas $\left\{X_{t}\right\}$ y $\left\{Z_{t}\right\}$ está dada por

$$
p\left(\mathbf{x}, \mathbf{z} \mid \boldsymbol{\theta}_{x}, \boldsymbol{\theta}_{z}\right)=p\left(\mathbf{x} \mid \mathbf{z}, \boldsymbol{\theta}_{x}, \boldsymbol{\theta}_{z}\right) p\left(\mathbf{z} \mid \boldsymbol{\theta}_{x}, \boldsymbol{\theta}_{z}\right)
$$

donde $\boldsymbol{\theta}_{x}$ contiene los parámetros no estructurales del modelo TAR, y $\theta_{z}$ contiene los parámetros concernientes al proceso $\left\{Z_{t}\right\}$.

En la anterior expresión para la función de verosimilitud, se tiene que

$$
p\left(\mathbf{z} \mid \boldsymbol{\theta}_{x}, \boldsymbol{\theta}_{z}\right)=f\left(\mathbf{z}_{p} \mid \boldsymbol{\theta}_{z}\right) f\left(z_{p+1} \mid \mathbf{z}_{p}, \boldsymbol{\theta}_{z}\right) \cdots p\left(z_{T} \mid \mathbf{z}_{T-1}, \boldsymbol{\theta}_{z}\right)
$$

$\operatorname{con} \mathbf{z}_{t}=\left(z_{t}, z_{t-1}, \cdots, z_{t-p+1}\right)^{\prime}, \mathrm{y}$

$$
p\left(\mathbf{x} \mid \mathbf{z}, \boldsymbol{\theta}_{x}, \boldsymbol{\theta}_{z}\right)=p\left(x_{k+1} \mid \mathbf{x}_{k}, \mathbf{z}, \boldsymbol{\theta}_{x}, \boldsymbol{\theta}_{z}\right) \cdots p\left(x_{T} \mid x_{T-1}, \cdots, x_{1}, \mathbf{z}, \boldsymbol{\theta}_{x}, \boldsymbol{\theta}_{z}\right)
$$

Si $e_{t} \sim t_{n}$, entonces para cada $t=k+1, \cdots, T, x_{t} \mid x_{t-1}, \cdots, x_{1}$ se distribuye como una variable $t_{n}$ multiplicado por $h^{\left(j_{t}\right)}$ y sumado por $a_{0}^{\left(j_{t}\right)}+\sum_{i=1}^{k_{j_{t}}} a_{i}^{\left(j_{t}\right)} x_{t-i}$. 
De esta forma

$$
\begin{aligned}
& p\left(x_{t} \mid x_{t-1}, \cdots, x_{1}, \mathbf{z}, \boldsymbol{\theta}_{x}, \boldsymbol{\theta}_{z}\right) \\
& =\frac{\Gamma\left(\frac{n+1}{2}\right)}{\sqrt{n \pi} \Gamma\left(\frac{n}{2}\right)} \frac{1}{h^{\left(j_{t}\right)}}\left(1+\frac{\left[x_{t}-a_{0}^{\left(j_{t}\right)}-\sum_{i=1}^{k_{j}} a_{i}^{\left(j_{t}\right)} x_{t-i}\right]^{2}}{\left(h^{\left(j_{t}\right)}\right)^{2} n}\right)^{-\frac{n+1}{2}}
\end{aligned}
$$

donde $\left\{j_{t}\right\}$ es la realización del proceso de indicadores $\left\{J_{t}\right\}$ definido como $J_{t}=j$ si $Z_{t} \in R_{j}$, para algún $j=1, \cdots, l$.

Por consiguiente se tiene que

$$
p\left(\mathbf{x} \mid \mathbf{z}, \boldsymbol{\theta}_{x}, \boldsymbol{\theta}_{z}\right)=\left[\frac{\Gamma\left(\frac{n+1}{2}\right)}{\sqrt{n \pi} \Gamma\left(\frac{n}{2}\right)}\right]^{T-k} \prod_{t=k+1}^{T}\left[h^{\left(j_{t}\right)}\right]^{-1} \prod_{t=k+1}^{T}\left(1+\frac{e_{t}^{2}}{n}\right)^{-\frac{n+1}{2}}
$$

$\operatorname{con} e_{t}=\frac{1}{h^{\left(j_{t}\right)}}\left(x_{t}-a_{0}^{\left(j_{t}\right)}-\sum_{i=1}^{k_{j_{t}}} a_{i}^{\left(j_{t}\right)} x_{t-i}\right)$.

Finalmente, nótese que en la función de verosimilitud (2), el término $f\left(\mathbf{z} \mid \boldsymbol{\theta}_{x}, \boldsymbol{\theta}_{z}\right)$ no contiene los parámetros de interés $\boldsymbol{\theta}_{x}$. De esta forma, en el desarrollo bayesiano del próximo capítulo, la función de verosimilitud se reducirá a $f\left(\mathbf{x} \mid \mathbf{z}, \boldsymbol{\theta}_{x}, \boldsymbol{\theta}_{z}\right)$.

\section{Estimación bayesiana de los parámetros no es- tructurales}

En esta parte, se encuentra la distribución a posteriori de los parámetros no estructurales, y se implementa el muestreador de Gibbs para obtener la estimación bayesiana de estos parámetros.

\subsection{Distribución a posteriori de los parámetros no estructu- rales}

Para encontrar la distribución a posteriori de los parámetros no estructurales, se necesita la función de verosimilitud del modelo encontrada anteriormente y la distribución a priori de los parámetros.

Sea $\boldsymbol{\theta}_{j}=\left(a_{0}^{(j)}, a_{1}^{(j)}, \cdots, a_{k_{j}}^{(j)}\right)^{\prime}$, para $j=1, \cdots, l$, y $\boldsymbol{\theta}=\left(\boldsymbol{\theta}_{1}^{\prime}, \cdots, \boldsymbol{\theta}_{l}^{\prime}\right)^{\prime}$, la distribución a priori para $\boldsymbol{\theta}_{j}$ es una distribución normal multivariante, esto es, $\boldsymbol{\theta}_{j} \sim$ $N\left(\boldsymbol{\theta}_{0, j}, \mathbf{V}_{0, j}^{-1}\right)$. En la mayoría de los casos se toma $\boldsymbol{\theta}_{0, j}=\mathbf{0}, \mathbf{V}_{0, j}$ diagonal con valores pequeños en la diagonal, representando la falta de certeza en la información a priori. Usando la anterior distribución a priori y la función de verosimilitud en (3). 
Resultado 3.1. Para cada $j=1, \cdots$, l, la distribución condicional de $\boldsymbol{\theta}_{j}$ dados los demás parámetros no estructurales $\boldsymbol{\theta}_{i}, i \neq j, \mathbf{h}, \boldsymbol{\theta}_{z}$, y $n$ está dada por

$$
\begin{aligned}
& p\left(\boldsymbol{\theta}_{j} \mid \boldsymbol{\theta}_{i}, i \neq j, \mathbf{h}, \mathbf{x}, \mathbf{z}, n\right) \\
& \propto \prod_{\left\{t: j_{t}=j\right\}}\left(1+\frac{\left[x_{t}-a_{0}^{(j)}-\sum_{i=1}^{k_{j}} a_{i}^{(j)} x_{t-i}\right]^{2}}{\left(h^{(j)}\right)^{2} n}\right)^{-\frac{n+1}{2}} \\
& \exp \left\{-\frac{1}{2}\left(\boldsymbol{\theta}_{j}-\boldsymbol{\theta}_{0, j}\right)^{\prime} \mathbf{V}_{0, j}\left(\boldsymbol{\theta}_{j}-\boldsymbol{\theta}_{0, j}\right)\right\}
\end{aligned}
$$

Prueba 3.1. Es claro que

$$
p\left(\boldsymbol{\theta}_{j} \mid \boldsymbol{\theta}_{i}, i \neq j, \mathbf{h}, \mathbf{x}, \mathbf{z}, n\right) \propto p(\mathbf{x} \mid \boldsymbol{\theta}, \mathbf{z}, \mathbf{h}, n) p\left(\boldsymbol{\theta}_{j}\right)
$$

donde

$$
\begin{aligned}
& p(\mathbf{x} \mid \boldsymbol{\theta}, \mathbf{z}, \mathbf{h}, n) \\
= & {\left[\frac{\Gamma\left(\frac{n+1}{2}\right)}{\sqrt{n \pi} \Gamma\left(\frac{n}{2}\right)}\right]^{T-k} \prod_{t=k+1}^{T}\left[h^{\left(j_{t}\right)}\right]^{-1} \prod_{t=k+1}^{T}\left(1+\frac{e_{t}^{2}}{n}\right)^{-\frac{n+1}{2}} } \\
\propto & \prod_{t=k+1}^{T}\left(1+\frac{\left[x_{t}-a_{0}^{\left(j_{t}\right)}-\sum_{i=1}^{k_{j}} a_{i}^{\left(j_{t}\right)} x_{t-i}\right]^{2}}{\left(h^{\left(j_{t}\right)}\right)^{2} n}\right)^{-\frac{n+1}{2}} \\
\propto & \prod_{\left\{t: j_{t}=j\right\}}\left(1+\frac{\left[x_{t}-a_{0}^{(j)}-\sum_{i=1}^{k_{j}} a_{i}^{(j)} x_{t-i}\right]^{2}}{\left(h^{(j)}\right)^{2} n}\right)^{-\frac{n+1}{2}}
\end{aligned}
$$

donde la última ecuación se tiene después de eliminar los términos que depende de $\boldsymbol{\theta}_{i}$ con $i \neq j$, puesto que en la densidad de interés $p\left(\boldsymbol{\theta}_{j} \mid \boldsymbol{\theta}_{i}, i \neq j, \mathbf{h}, \mathbf{x}, \mathbf{z}, n\right)$, las cantidades $\boldsymbol{\theta}_{i}$ con $i \neq j$ se consideran constantes.

De esta forma,

$$
\begin{aligned}
& p\left(\boldsymbol{\theta}_{j} \mid \boldsymbol{\theta}_{i}, i \neq j, \mathbf{h}, \mathbf{x}, \mathbf{z}\right) \\
& \propto \prod_{\left\{t: j_{t}=j\right\}}\left(1+\frac{\left[x_{t}-a_{0}^{(j)}-\sum_{i=1}^{k_{j}} a_{i}^{(j)} x_{t-i}\right]^{2}}{\left(h^{(j)}\right)^{2} n}\right)^{-\frac{n+1}{2}} \\
& \exp \left\{-\frac{1}{2}\left(\boldsymbol{\theta}_{j}-\boldsymbol{\theta}_{0, j}\right)^{\prime} \mathbf{V}_{0, j}\left(\boldsymbol{\theta}_{j}-\boldsymbol{\theta}_{0, j}\right)\right\}
\end{aligned}
$$

Nótese que (1) en el anterior resultado, el único componente de $\mathbf{h}$ que afecta la distribución a posteriori condicional de $\boldsymbol{\theta}_{j}$ es el componente $h^{(j)} ;(2)$ la anterior 
densidad condicional no corresponde a una densidad conocida, y por consiguiente, se deben utilizar métodos de simulación como el método de grille1 por su simplicidad.

Ahora, con respecto a los parámetros $h^{(1)}, \cdots, h^{(l)}$, la distribución a priori para $\left(h^{(j)}\right)^{2}$ es una distribución inversa Gamma con parámetro de forma $\alpha$ y parámetro de escala $\beta$, (Inverse - Gamma $(\alpha, \beta))$, es decir,

$$
p\left(\left(h^{(j)}\right)^{2}\right) \propto\left(h^{(j)}\right)^{-2 \alpha-2} \exp \left\{-\beta /\left(h^{(j)}\right)^{2}\right\} I_{(0, \infty)}\left(\left(h^{j}\right)^{2}\right)
$$

Usando esta distribución a priori de $\left(h^{(j)}\right)^{2}$, tenemos el siguiente resultado para la distribución a posteriori condicional de $\left(h^{(j)}\right)^{2}$

Resultado 3.2. Para cada $j=1, \cdots, l$, la distribución condicional de $\left(h^{(j)}\right)^{2}$ dados los parámetros no estructurales, $\boldsymbol{\theta}_{j}, j=1, \cdots, l, \mathbf{h}$ y $\boldsymbol{\theta}_{z}$ está dada por

$$
\begin{aligned}
& p\left(\left(h^{(j)}\right)^{2} \mid \boldsymbol{\theta}_{1}, \cdots, \boldsymbol{\theta}_{l}, \mathbf{x}, \mathbf{z}, n\right) \\
\propto & \prod_{\left\{t: j_{t}=j\right\}}\left(1+\frac{\left[x_{t}-a_{0}^{(j)}-\sum_{i=1}^{k_{j}} a_{i}^{(j)} x_{t-i}\right]^{2}}{\left(h^{(j)}\right)^{2} n}\right)^{-\frac{n+1}{2}}\left(h^{(j)}\right)^{-2 \alpha-2-n_{j}} \exp \left\{-\beta /\left(h^{(j)}\right)^{2}\right\}
\end{aligned}
$$

donde $n_{j}=\#\left\{t: j_{t}=j\right\}$.

Demostración.

$$
\begin{aligned}
& p\left(\left(h^{(j)}\right)^{2} \mid \boldsymbol{\theta}_{1}, \cdots, \boldsymbol{\theta}_{l}, \mathbf{x}, \mathbf{z}, n\right) \\
\propto & p(\mathbf{x} \mid \boldsymbol{\theta}, \mathbf{z}, \mathbf{h}, n) p\left(\left(h^{(j)}\right)^{2}\right) \\
\propto & \prod_{t=k+1}^{T}\left(h^{\left(j_{t}\right)}\right)^{-1}\left(1+\frac{\left[x_{t}-a_{0}^{\left(j_{t}\right)}-\sum_{i=1}^{k_{j t}} a_{i}^{\left(j_{t}\right)} x_{t-i}\right]^{2}}{\left(h^{\left(j_{t}\right)}\right)^{2} n}\right)^{-\frac{n+1}{2}} \\
\times & \left(h^{(j)}\right)^{-2 \alpha-2} \exp \left\{-\beta /\left(h^{(j)}\right)^{2}\right\} \\
\propto & \prod_{\left\{t: j_{t}=j\right\}}\left(1+\frac{\left[x_{t}-a_{0}^{(j)}-\sum_{i=1}^{k_{j}} a_{i}^{(j)} x_{t-i}\right]^{2}}{\left(h^{(j)}\right)^{2} n}\right)^{-\frac{n+1}{2}}\left(h^{(j)}\right)^{-2 \alpha-2-n_{j}} \exp \left\{-\beta /\left(h^{(j)}\right)^{2}\right\}
\end{aligned}
$$

Nótese que en el anterior resultado, el único componente de $\boldsymbol{\theta}_{1}, \cdots, \boldsymbol{\theta}_{l}$ que afecta la distribución condicional a posteriori de $\left(h^{(j)}\right)^{2}$ es el componente $\boldsymbol{\theta}_{j}$. Adicionalmente, la anterior densidad tampoco tiene una forma cerrada y se puede muestrear con el método de la grilla.

\footnotetext{
${ }^{1}$ Para mayores detalles y ejemplos sobre este método, el lector puede consultar la página http://www.gutierrezandres. com/?s=grilla
} 
Finalmente, para implementar el muestreador de Gibbs, resta encontrar la distribución condicional a posteriori del grado de libertad del proceso de ruido $n$. Para la distribución a priori de $n$, inicialmente se pensó en una distribución uniforme discreta sobre el conjunto $\left\{2, \cdots, n_{\max }\right\}$; sin embargo, se vio que el muestreador de Gibbs resultante de esta distribución a priori arroja una estimación de $n$ muy por debajo del valor real.

Por esta razón, la distribución a priori que se asigna a $n$ es una distribución Gamma, siguiendo a la sugerencia de Watanabe (2001), puesto que en la distribución $\operatorname{Gamma}\left(\alpha^{\prime}, \beta^{\prime}\right)$, la media está dada por $\alpha^{\prime} \beta^{\prime}$ y la varianza $\alpha^{\prime} \beta^{\prime 2}$, y en la práctica se puede escoger los parámetros a priori de $n$ de tal forma que la media y la varianza a priori coincidan con el conocimiento previo del investigador, o escoger los parámetros a priori para obtener una varianza a priori grande representando la incertidumbre o la falta de información a priori acerca de $n$. De esta forma la a priori de $n$ está dada por

$$
p(n) \propto n^{\alpha^{\prime}-1} \exp \left\{-n / \beta^{\prime}\right\}
$$

Con la anterior función de densidad a priori de $n$, se encuentra la siguiente distribución condicional a posteriori de $n$

Resultado 3.3. La distribución a posteriori del grado de libertad del proceso del ruido $n$ condicionando en los demás parámetros no estructurales está dada por

$$
\begin{aligned}
& p\left(n \mid \boldsymbol{\theta}_{1}, \cdots, \boldsymbol{\theta}_{l}, \mathbf{h}, \mathbf{x}, \mathbf{z}\right) \\
\propto & {\left[\frac{\Gamma\left(\frac{n+1}{2}\right)}{\Gamma\left(\frac{n}{2}\right)}\right]^{T-k} \prod_{t=k+1}^{T}\left(1+\frac{\left[x_{t}-a_{0}^{\left(j_{t}\right)}-\sum_{i=1}^{k_{j_{t}}} a_{i}^{\left(j_{t}\right)} x_{t-i}\right]^{2}}{\left(h^{\left(j_{t}\right)}\right)^{2} n}\right) } \\
\times & n^{-\frac{1}{2}(T-k)+\alpha^{\prime}-1} \exp \left\{-n / \beta^{\prime}\right\}
\end{aligned}
$$

Demostración.

$$
\begin{aligned}
& p\left(n \mid \boldsymbol{\theta}_{1}, \cdots, \boldsymbol{\theta}_{l}, \mathbf{h}, \mathbf{x}, \mathbf{z}\right) \\
\propto & p(\mathbf{x} \mid \boldsymbol{\theta}, \mathbf{z}, \mathbf{h}, n) \pi(n) \\
\propto & {\left[\frac{\Gamma\left(\frac{n+1}{2}\right)}{\sqrt{n} \Gamma\left(\frac{n}{2}\right)}\right]^{T-k} \prod_{t=k+1}^{T}\left(1+\frac{\left[x_{t}-a_{0}^{\left(j_{t}\right)}-\sum_{i=1}^{k_{j}} a_{i}^{\left(j_{t}\right)} x_{t-i}\right]^{2}}{\left(h^{\left(j_{t}\right)}\right)^{2} n}\right)^{-\frac{n+1}{2}} n^{k-1} \exp \{-n / \theta\} } \\
\propto & {\left[\frac{\Gamma\left(\frac{n+1}{2}\right)}{\Gamma\left(\frac{n}{2}\right)}\right]^{T-k} \prod_{t=k+1}^{T}\left(1+\frac{\left[x_{t}-a_{0}^{\left(j_{t}\right)}-\sum_{i=1}^{k_{j}} a_{i}^{\left(j_{t}\right)} x_{t-i}\right]^{2}}{\left(h^{\left(j_{t}\right)}\right)^{2} n}\right)^{-\frac{n+1}{2}} } \\
\times & n^{-\frac{1}{2}(T-k)+\alpha^{\prime}-1} \exp \left\{-n / \beta^{\prime}\right\}
\end{aligned}
$$


Utilizando las tres anteriores densidades condicionales a posteriori, se puede implementar un muestreador de Gibbs que se describe a continuación.

\subsection{Muestreador de Gibbs para estimar parámetros}

Los pasos del muestreador de Gibbs para estimar los parámetros no estructurales del régimen $j: \boldsymbol{\theta}_{j}, h^{(j)}$ para $j=1, \cdots, l$ y $n$ son como siguen:

(1) Fijar valores iniciales para $\boldsymbol{\theta}_{j}$, se denotan por $\boldsymbol{\theta}_{j, 0}$,

(2) Fijar un valor inicial para $h^{(j)}$ y se denota por $h_{0}^{(j)}$,

(3) Fijar un valor inicial para $n$ denotada por $n_{0}$,

(4) Para la iteración $g$, con $g=1, \cdots, G$, donde $G$ es la longitud de la cadena, se simula un valor para el vector $\boldsymbol{\theta}_{j}$ a partir de la densidad condicional $p\left(\boldsymbol{\theta}_{j} \mid \boldsymbol{\theta}_{i, g-1}, i \neq j, h_{g-1}^{j}, \boldsymbol{\alpha}, \mathbf{x}, \mathbf{z}, n_{g-1}\right)$, el valor obtenido se denota por $\boldsymbol{\theta}_{j, g}$,

(5) Se simula un valor para $h^{(j)}$ a partir de la densidad condicional $p\left(\left(h^{(j)}\right)^{2} \mid \boldsymbol{\theta}_{j, g}, \mathbf{x}, \mathbf{z}, n_{g-1}\right)$, el valor obtenido se denota por $h_{g}^{(j)}$,

(6) Se simula un valor para $n$ de la densidad condicional $p\left(n \mid \boldsymbol{\theta}_{1, g}, \cdots, \boldsymbol{\theta}_{l, g}, \mathbf{h}_{g}, \mathbf{x}, \mathbf{z}\right)$

A continuación se presenta un ejemplo simulado para comprobar empíricamente la eficacia del anterior muestreador de Gibbs.

\section{Ejemplo simulado}

Se simuló una serie de tiempo $\left\{x_{t}\right\}$ de longitud 300 siguiendo el modelo

$$
X_{t}= \begin{cases}1+0.5 X_{t-1}-0.3 X_{t-2}+e_{t} & \text { si } Z_{t} \leq 0 \\ -0.5-0.7 X_{t-1}+1.5 e_{t} & \text { si } Z_{t}>0\end{cases}
$$

con $\left\{e_{t}\right\} \sim_{\text {iid }} t_{5}, Z_{t}=0.5 Z_{t-1}+\epsilon_{t} \mathrm{y} \epsilon_{t} \sim R B(0,1)$. Las series simuladas $\left\{x_{t}\right\} \mathrm{y}$ $\left\{z_{t}\right\}$ se pueden ver en la figura 1 .

Las distribuciones a priori para los parámetros no estructurales son: distribución $N(0,10)$ para los coeficientes autorregresivos $a_{i}^{j}$ con $i=1, \cdots, k_{j}$ y $j=1,2$; distribución Inverse - $\operatorname{Gamma}(2,3)$ para las ponderaciones de varianza $h^{(1)}$ y $h^{(2)}$; distribución $\operatorname{Gamma}(2,5)$ para el grado de libertad $n$. En este caso, la media a priori para $n$ es de 10 y la varianza a priori es de 50, lo cual se puede considerar como una distribución a priori poco informativat.

\footnotetext{
${ }^{2}$ Se vio en simulaciones adicionales, que no hay diferencia significativa entre las estimaciones de $n$ al poner una varianza a priori más grande que 50; pero al poner la varianza a priori más pequeña, como 10, la estimación obtenida de $n$ se acercará más a la media a priori 10 . Por esta razón, se escogió el valor de 50 para la varianza a priori.
} 

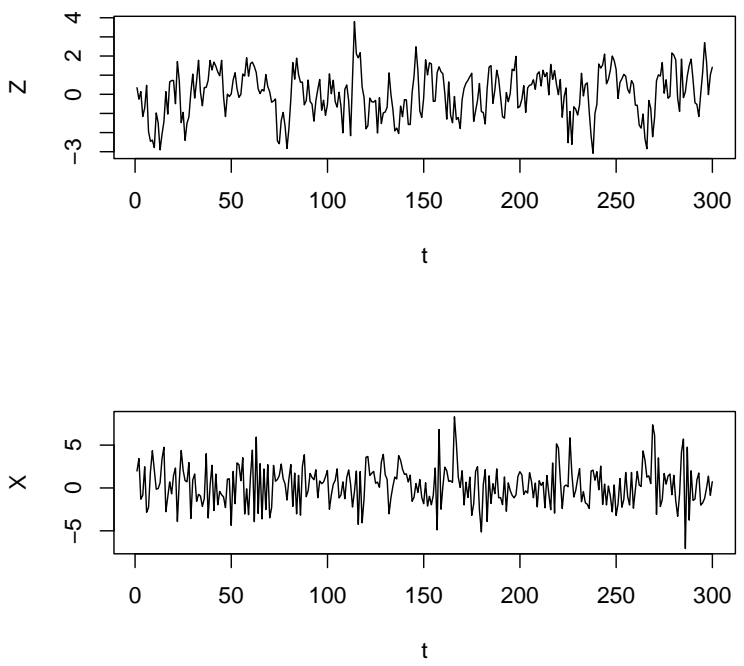

Figura 1: Serie $\left\{x_{t}\right\}$ y $\left\{z_{t}\right\}$ simulados según el modelo (4).

Tabla 1: Estimación e intervalos de credibilidad del $95 \%$ para los parámetros no estructurales estimados del ejemplo simulado 1.

\begin{tabular}{|c|ccc|c|}
\hline Régimen & \multicolumn{3}{|c|}{$a_{i}^{(j)}$} & $h^{(j)}$ \\
\hline 1 & 0.88 & 0.52 & -0.33 & 1.13 \\
& $(0.66,1.11)$ & $(0.42,0.62)$ & $(-0.42,-0.23)$ & $(0.95,1.32)$ \\
\hline 2 & -0.53 & -0.62 & 1.38 \\
& $(-0.78,-0.33)$ & $(-0.72,-0.51)$ & & $(1.16,1.62)$ \\
\hline
\end{tabular}

Los parámetros estructurales se asumen conocidos, después de 500 iteraciones del muestreador de Gibbs, se toma la segunda mitad de la cadena para calcular las estimaciones que bajo la función de pérdida cuadrática, corresponden a la esperanza de las distribuciones a posteriori. Las estimaciones de los parámetros no estructurales se muestran en la tabla 1, donde se puede ver que las estimaciones puntuales son buenas, y todos los intervalos de credibilidad del $95 \%$ contienen a los parámetros verdaderos.

Con respecto al grado de libertad $n$, la estimación está dada por 4.9988, con un intervalo de credibilidad del $95 \%$ de $(3.12,9.14)$. Es importante resaltar que las anteriores estimaciones de los parámetros se mantienen si se varía el valor de la media a priori de $n$ manteniendo una varianza a priori grande. Finalmente las gráficas CUSUM y CUSUMSQ en la figura 2 muestran que ajuste del modelo estimado, en general, es bueno. 
CUSUM

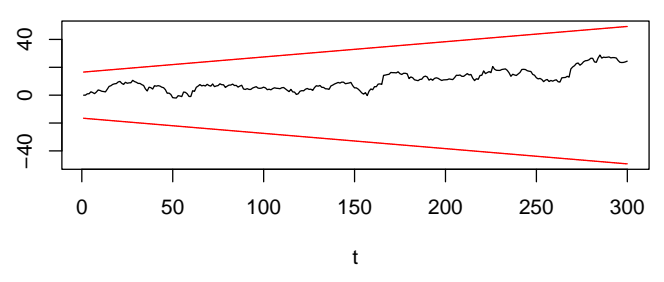

CUSUMSQ

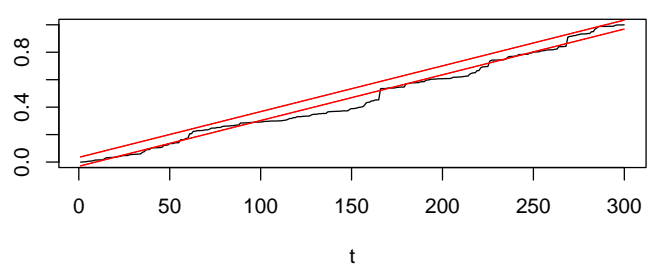

Figura 2: Gráficas CUSUM y CUSUMSQ para los residuos del modelo estimado.

\section{Discusión}

En este artículo se desarrolló una metodología bayesiana para estimar un modelo TAR con ruido $t$ una vez los parámetros estructurales hayan sido identificados. Con el ejemplo simulado se vio que las estimaciones obtenidas son buenas; sin embargo, la computación de estas estimaciones demanda bastante tiempo, y esta dificultad se debe a que las distribuciones condicionales a posteriori de los parámetros no son conjugadas y no tienen una forma cerrada. En este artículo se usó el método de la grilla para obtener muestras de estas densidades condicionales; no obstante, otros métodos como el de Metropolis Hasting también podrían ser utilizados. Futuras investigaciones podrían basarse en la identificación del modelo TAR estimando los parámetros estructurales y el cálculo del pronóstico del modelo.

Este artículo hace parte de la tesis doctoral en desarrollo de la autora bajo la dirección del profesor Fabio H. Nieto del Departamento de Estadística, Universidad Nacional de Colombia.

Recibido: 20 de septiembre de 2011

Aceptado: 1 de noviembre de 2011

Comunicaciones en Estadística, diciembre 2011, Vol. 4, No. 2 


\section{Referencias}

Moreno, E. C. (2010), Modelos tar en series de tiempo financieras., Master's thesis, Universidad Nacional de Colombia.

Nieto, F. H. (2005), 'Modeling bivariate threshold autoregressive processes in the presence of missing data.', Communications in Statistics, Theory and Methods. 34, $905-930$.

Nieto, F. H. (2008), 'Forecasting with univariate tar models.', Statistical Methodology. 5, $263-276$.

Tong, H. (1978), 'On a thereshold model.', Pattern Recognition and Signal Processing, Ed. C. H. Chen. Sijthoff $\mathcal{E}$ Noordhoff, Netherlands. NATO ASI Series E: Applied Sc. 29, $575-586$.

Tsay, R. S. (1998), 'Testing and modeling multivariate threshold models.', J. Amer. Statist. Assoc. 93, $1188-1202$.

Watanabe, T. (2001), 'On sampling the degree-of-freedom of student's- $t$ disturbaces', Satisticas \&3 Probability Letters 52, 177 - 181. 